\title{
REHABILITATION OF PARTIALLY \\ EDENTULOUS PATIENT USING REMOVABLE PARTIAL DENTURE WITH ESTHETIC RETAINERS - A CASE REPORT
}

Dr.Ranjith.R, Dr.Devi Parameswari.B, Dr. Annapoorni.H

Department of Prosthodontics, Faculty of Dentistry,

Meenakshi Academy of Higher Education and Research Institute, Chennai, Tamil Nadu, India.

\section{To access \& cite this article}

Website: jidam.idamadras.com

\section{Address for correspondence:}

Dr.B.Devi Parameswari, MDS

Associate Professor,

Department of Prosthodontics,

Faculty of Dentistry, Meenakshi Academy

of Higher Education and Research Institute,

Chennai, Tamil Nadu, India.

Email id: drdeviparameswari@gmail.com

\section{ABSTRACT}

The treatment options available for esthetic and functional rehabilitation of partially edentulous patients are fixed partial dentures(FPD) and removable partial dentures, where fixed could be conventional FPDs or implant FPDs. In some cases, where fixed partial dentures are not possible, removable partial dentures may be the only choice to the patients. Location of clasps in the esthetic zone of the removable cast partial dentures, is not aesthetically pleasant to the patients. Among many material choices for removable denture fabrications, flexible partial dentures provide superior esthetics especially for those patients who are concerned more about esthetics.

KEYWORDS: Flexible denture, Dent flex, Nylon, Acrylic clasp, Flipper.

Received : : 18.05.2020

Accepted : : 13.06.2020

Published : 27.06.2020 


\section{INTRODUCTION:}

Prosthetic rehabilitation of partially edentulous patient restores patient's masticatory function and esthetics. Fabrication of a metal framework of conventional removable partial denture (RPDs) uses metal retentive clasps. On smiling, these metal clasps are usually visible which cause dissatisfaction to the patient which psychologically affect the patient's social behaviour due to unpleasant esthetics. ${ }^{1}$ The introduction of acrylic polymers and cobalt chromium alloys type of removable partial dentures have gained popularity among dentists these days. Many years ago, removable partial denture was chosen by patients for their low cost and good physiology. Modern era has led to the evolvement of the removable partial denture from traditional hard fitting denture bases to soft dentures by using nylon based thermoplastic material. So, they have become an excellent alternative to traditional removable partial dentures which have many problems like loss of chewing efficiency, hard denture bases and less retention. ${ }^{2}$ These flexible dentures provide good function and esthetics, comfort and strength. The flexible nylon resin has simple design, a built- in stress breaker which provide better stress distribution and a superior function. So flexible denture has become a good option not only for dentists, but also for patients. ${ }^{3}$

This article describes about the restoration of partially edentulous patients using a flexible removable partial denture.

\section{CASE REPORT:}

A 65 year old female patient reported to the Department of Prosthodontics with missing maxillary and mandibular premolar and molar teeth bilaterally. On intra-oral examination, the patient had missing 14, 16, 21, 22, 23, 24 and 26 (Kennedy's class III modification 3) (Fig 1), 36 and 44 (Kennedy's class III modification 1) (Fig 2) with facial view in occlusion as in Fig 3. After complete examination, maxillary flexible denture prosthesis was planned as patient was concerned only about the maxilla and patient did not want mandibular prosthesis.

\section{PROCEDURE:}

were made(Dentsply Zelgan 2002 Alginate) and casts were made with Type III dental stone (Gem stone dental stone plaster type-III). Interocclusal record was done. Casts were mounted using the interocclusal record on the Hanau articulator. Shade selection was done and artificial acrylic resin teeth (SR Vivodent, Ivoclar vivadent) were arranged. Wax try-in was done to the patient, acrylic clasp extension was placed on the right central incisor and denture was processed using the injection moulding technique (Dentcare Flex - Dentcare dental lab, India). Then the final denture was finished, polished and inserted. Figures 4 and 5 show side and facial view after denture insertion. Occlusion was evaluated and adjusted.

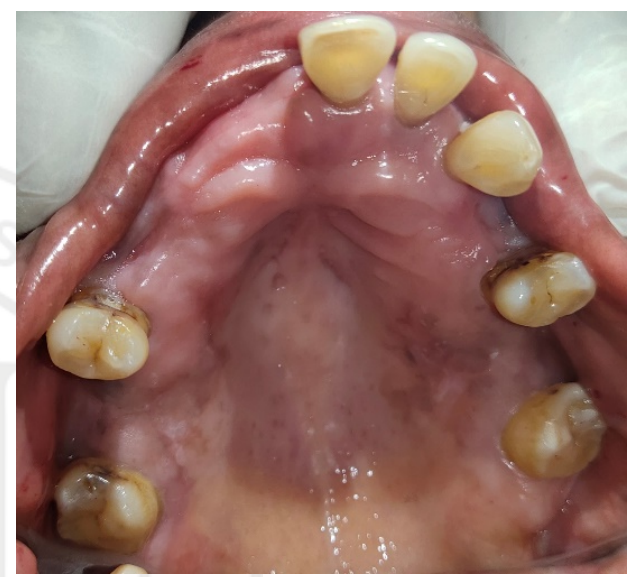

Fig 1: Intra-Oral View of Maxilla

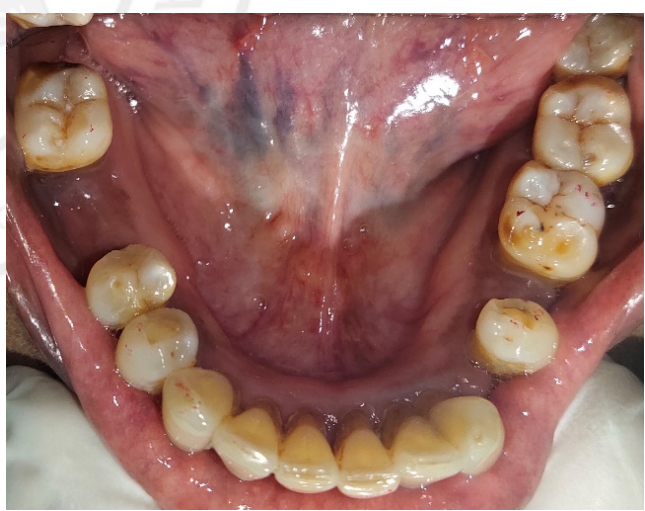

Fig 2: Intra-Oral View of Mandible

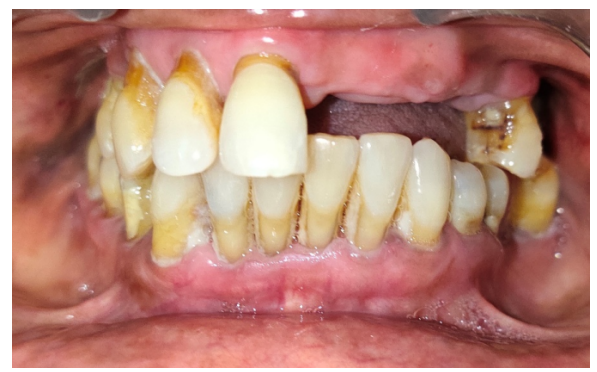

Fig 3: Facial View

Maxillary and mandibular alginate impressions 


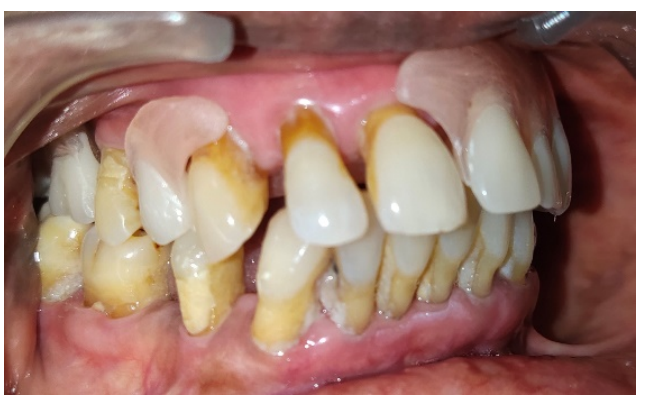

Fig 4: Postop Intra-oral side view

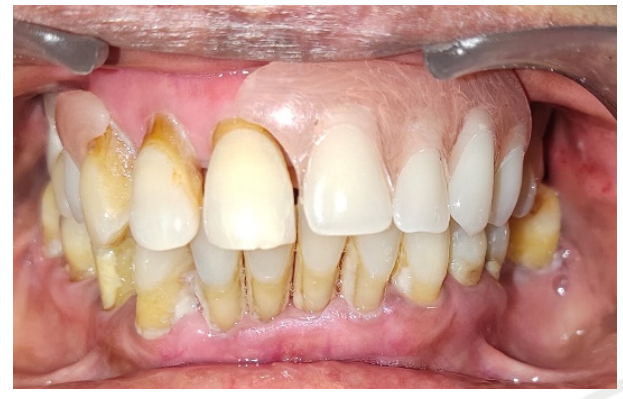

Fig 5: Postop Intra-oral facial view

are derived from dicarboxylic acid, diamine, amino acid and lactams. ${ }^{5}$ The commonest technique used for fabrication of flexible denture base prosthesis is the Injection-molding technique. ${ }^{6}$ Flexible denture prosthesis has many advantages from others as it requires minimum or no mouth preparation, resistant to fracture, match with natural tissues, good retention, comfortable for patient as it is thin and lightweight. Acrylic resin teeth do not tend to bond chemically with a flexible denture base resin and are retained by making $\mathrm{T}$ shaped holes into which the resin flows to retain teeth mechanically. ${ }^{7}$

Thin finger like extension of the flexible partial denture extends into the undercuts that act like a clasp. They can be used in patients having gingival recession and in patients who are allergic to acrylic. ${ }^{2}$ However, they are contraindicated in patients with insufficient inter-arch space i.e., less than $4 \mathrm{~mm}$ space, and with prominent residual ridges where there is less space for labial placement of teeth because Tshape holes are necessary for mechanical retention of teeth to denture base, and with flat-flabby ridges with poor soft tissue support which require more rigid prosthesis. $^{3}$

Removable partial denture is commonly used for treating those patients where multiple teeth are missing and where conventional fixed partial dentures and implant supported prosthesis are not possible. For more than ten years, the removable cast partial denture is being used in the treatment of partially edentulous patients and its construction involves the use of cast metal alloy, acrylic resin and/ or thermoplastic resins. Cast metal alloy consists of a metal base with acrylic teeth linked to it and metal retentive clasp aids in holding the cast partial denture in position. Metal clasps give metallic appearance in the esthetic zone which is not acceptable by many patients, especially those who are concerned about their esthetics. ${ }^{4}$

Acrylic resin prosthesis is also known as temporary or interim removable partial denture or a 'Flipper'. It can function as a space maintainer and also can be used to restore the form and function of the tooth until the definitive prosthesis is fabricated. Flexible denture materials are superpolyamides belonging to the family of nylon and are available in the form of granules that are packed in cartridges of varying sizes and first introduced by the name of valplast and flexiplast since 1956. These resins

One drawback of flexible prosthesis is that they are difficult to repair, reline and rebase. They are prone to staining from tea and/or coffee if it is not polished properly and cleaned by the patient on a regular basis. The esthetics and cleanliness of the prosthesis can be maintained by advising the patient to practice good oral hygiene and clean his/her prosthesis regularly after every meal and it should be removed during brushing of natural teeth to avoid roughening of the prosthesis. A commercially available denture cleanser (Val-Clean ${ }^{\circledR}$ ) where denture is soaked for $10-15$ minutes daily can be advised.

\section{CONCLUSION:}

Flexible partial denture is a good treatment option for partially edentulous patients, who are concerned about esthetics. In order to reduce staining of the prosthesis, proper care of the prosthesis is required, as it affects the esthetics of the prosthesis as time passes by. The fabrication depends on the clinician's skill in selection of the type of the prosthesis required for the patient. Flexible dentures while previously used were selected by only a handful 
of patients and the clinician has gained its ground to become an effective and often demanded treatment option.

FINANCIAL SUPPORT AND

SPONSORSHIP:

Nil

\section{CONFLICTS OF INTEREST:}

There are no conflicts of interest.

\section{REFERENCES:}

1. Chu $\mathrm{CH}$, Chow TW. Esthetic designs of removable partial dentures. Gen Dent 2003 Aug;51(4):322-4.

2. GitanjaliMago, Puneet Sharma. Flexible Denture: A Hope for Partial Edentulous Patient- A Case Report. Int Health Res J 2019 Nov 23;3(8):267-70.

3. Flexible denture for partially edentulous arches - case reports | International Journal of Recent Advances in Multidisciplinary Research (IJRAMR) [Internet]. [cited 2020 May 25]. Available from: https://www. ijramr.com/issue/flexible-denture-partiallyedentulous-arches-case-reports

4. Naylor WP, Manor RC. Fabrication of a flexible prosthesis for the edentulous scleroderma patient with microstomia. J Prosthet Dent 1983 Oct;50(4):536-8.

5. Yunus N, Rashid AA, Azmi LL, Abu-Hassan MI. Some flexural properties of a nylon denture base polymer. J Oral Rehabil 2005 Jan;32(1):65-71.

6. Phoenix RD, Mansueto MA, Ackerman NA, Jones RE. Evaluation of mechanical and thermal properties of commonly used denture base resins. J Prosthodont Off J Am CollProsthodont 2004 Mar;13(1):17-27.

7. Parvizi A, Lindquist $\mathrm{T}$, Schneider R, Williamson D, Boyer D, Dawson DV. Comparison of the dimensional accuracy of injection-molded denture base materials to that of conventional pressure-pack acrylic resin. J Prosthodont Off J Am CollProsthodont 2004 Jun;13(2):83-9. 